\title{
LA EXTRACCIÓN SELECTIVA DE VARA PARA USO HORTÍCOLA EN MÉXICO: IMPLICACIONES PARA LA CONSERVACIÓN DEL BOSQUE TROPICAL CADUCIFOLIO Y SUS RECURSOS
}

\author{
Humberto Rendón-Carmona ${ }^{1}$; Angelina Martínez-Yrízar ${ }^{2,4}$, J. Manuel Maass ${ }^{3}$, \\ Diego R. Pérez-Salicrup ${ }^{3}$ y Alberto Búrquez ${ }^{2}$ \\ ${ }^{1}$ Universidad Intercultural Indígena de Michoacán, Pátzcuaro, Michoacán, México \\ ${ }^{2}$ Instituto de Ecología, Universidad Nacional Autónoma de México, Hermosillo, Sonora, México \\ ${ }^{3}$ Centro de Investigaciones en Ecosistemas, Universidad Nacional Autónoma de México, Morelia, Michoacán, México \\ In Memoriam Dra. Cynthia A. Lindquist (1947-2011) \\ ${ }^{4}$ Autora para la correspondencia: angelina@unam.mx
}

\begin{abstract}
Resumen: Con base en una revisión bibliográfica, documentos oficiales e investigación propia, se analiza la extracción selectiva de vara (tutores de hortalizas) y los potenciales efectos de la remoción de este recurso forestal no maderable en la diversidad, estructura y regeneración del bosque tropical caducifolio de México. Las varas de mayor demanda pertenecen a un grupo de especies silvestres del género Croton (Euphorbiaceae), conocidas como "vara blanca", que son usadas como tutores en el cultivo de hortalizas. Tres sectores están involucrados en la extracción del recurso: cortadores, contratistas e intermediarios. Los intermediarios, encargados de la comercialización de la vara son los que obtienen los mayores ingresos. La regulación técnica que gobierna la extracción de vara en México es deficiente y carece de lineamientos básicos orientados hacia un aprovechamiento sostenible. Nuestros estudios en la región de Chamela, Jalisco, indican que la cosecha repetida de vara reduce la riqueza de especies, modifica la estructura de la comunidad y favorece la dominancia de Croton septemnervius a expensas de otras especies. Después del corte, la regeneración vegetativa de Croton es limitada, ya que el crecimiento de los rebrotes es lento, y los rebrotes y tocones sufren una alta mortalidad (> 70\%). Para lograr un manejo sostenible de este recurso forestal en el largo plazo, la calidad y el cumplimiento de la regulación técnica deben mejorar de forma inmediata. Asimismo, estudios interdisciplinarios del manejo de los bosques tropicales caducifolios que incorporen la interacción de aspectos ecológicos y socioeconómicos son urgentes.
\end{abstract}

Palabras clave: extracción selectiva, manejo sostenible, rebrote, regeneración natural.

\begin{abstract}
We review published, unpublished, and self-generated information related to the selective extraction of varas (stakes or support poles), and identify some potential effects of the removal of this non-timber forest product on the plant diversity, structure and regeneration of the tropical deciduous forests in Mexico. The varas in highest demand belong to a group of species of Croton (Euphorbiaceae), commercially known as vara blanca, which are widely used as stakes to support plants in horticultural fields. Three economic sectors are involved in the extraction of the vara resource: cutters, contractors, and intermediaries. The intermediaries, who are responsible for marketing the stakes, earn the largest revenues. Technical regulations in Mexico govern the extraction of varas, but they lack sound guidelines that will lead to sustainable use of the resource. Our studies in the Chamela region in Jalisco show that repeated extraction reduces species richness, modifies the structure of the plant community, and increases the relative dominance of Croton septemnervius at the expense of other tree species. Furthermore, after the stakes are cut for harvesting, its vegetative regeneration is limited. The stumps and sprouts exhibit high mortality $(>70 \%)$, and the sprouts that survive grow slowly. In order to achieve long-term sustainable management of this forest product, the quality, specificity, and enforcement of the management guidelines require immediate improvement. Also, interdisciplinary studies of tropical deciduous forests management encompassing ecological and socio-economic interactions are urgently needed.
\end{abstract}

Key words: natural regeneration, selective extraction, sprouting, sustainable management

$\mathbf{H}$ oy en día existe un gran interés a nivel mundial por identificar mecanismos efectivos de conservación de los bosques tropicales y de los servicios ecosistémicos que proveen. Esto se debe a que una gran parte de la biodiversidad y del carbono almacenado en los ecosistemas terrestres se encuentra en los bosques tropicales (55\% del carbono actual almacenado en los bosques del mundo, Pan et al., 2011), y a que muchos de estos bosques están sometidos a altas tasas de deforestación (FRA, 2010; Pan et al., 2011). Esta pérdida de áreas boscosas representa una amenaza para 
millones de personas en el mundo, principalmente para las poblaciones rurales de los países en desarrollo, cuyas necesidades de subsistencia diaria dependen en gran medida de la recolecta y uso de numerosos Productos Forestales No Maderables (PFNM; Marshall y Newton, 2003; Ticktin, 2004; Negi et al., 2011; Bowler et al., 2012).

De acuerdo con una evaluación de la FAO (FRA, 2000), México reconoce oficialmente el aprovechamiento de 250 tipos de PFNM, de los cuales 70 son comercialmente los más importantes, indicando que su explotación está sujeta a "algún tipo de control", y que sólo los productos de exportación o que se industrializan (p. ej., especias, resinas y látex) tienen una mayor regulación. Esta regulación es muy limitada, ya que se estima que en México se utilizan más de 5,000 plantas con diferentes fines, por lo que la gran mayoría de los PFNM no están sujetos a regulación oficial (Tapia-Tapia y Reyes-Chilpa, 2008). La falta de un marco de regulación apropiado que incluya a todas las especies aprovechadas, aunado al desconocimiento que se tiene sobre la diversidad de usos y comercialización de estos productos (CONABIO, 2008) agrava la problemática de conservación de los bosques y sus recursos.

Los PFNM son productos forestales (excluyendo la madera para la industria del aserrío) que se cosechan con propósitos comerciales o para ser utilizados por los propios recolectores. Estos productos incluyen plantas y sus partes como fibras, leña, tallos, cortezas, frutos y semillas que se usan con fines alimenticios, farmacéuticos, industriales, ornamentales y culturales (SEMARNAP, 1999; Tapia-Tapia y Reyes-Chilpa, 2008). Entre estos productos se encuentran los tallos recolectados para ser usados como estacas o tutores en los cultivos de hortalizas, llamados comúnmente, y de aquí en adelante en este artículo, como "varas". El uso de este recurso consiste en el corte selectivo de tallos de especies de plantas leñosas silvestres que sirven como tutores hortícolas para sostener el follaje, las ramas y los frutos de plantas cultivadas.

En México, el uso de varas como tutores en la horticultura tuvo su auge a partir de la segunda mitad del siglo pasado en algunos estados del norte y noroeste (Lindquist, 1999, 2000). Por la calidad de su madera, las varas con mayor demanda en México pertenecen al género Croton (Euphorbiaceae), un grupo de plantas nativas del Bosque Tropical Caducifolio (BTC) conocidas colectivamente como "vara blanca", muy apreciadas por el fuste recto, la madera dura y la resistencia a la pudrición. Dada la fuerte demanda de tutores, otras especies típicas del BTC, principalmente leguminosas, también se cortan pero con menor intensidad (Muro, 1987; López-Urquidez, 1997; Lindquist, 2000; RendónCarmona et al., 2009). A pesar de esta tradición de aprovechamiento forestal, no existe a la fecha un diagnóstico detallado sobre las especies proveedoras de vara y los niveles de extracción a los que están sometidos los BTC, ni estudios dirigidos a documentar los efectos del corte selectivo en la composición, estructura y dinámica de la vegetación de los sitios aprovechados. Esta información es fundamental para identificar objetivos de manejo congruentes con la conservación de los bosques y sus recursos (Lindquist, 2000).

El presente trabajo examina las implicaciones ecológicas y sociales de la extracción selectiva de vara para uso hortícola en México. Con base en una revisión bibliográfica y recopilación de documentos oficiales generados por el sector gubernamental, se presenta una síntesis del origen del aprovechamiento de este recurso del BTC y se mencionan las especies sujetas al corte selectivo. Asimismo, se explica el uso que se le da a la vara, se describen las tallas de los árboles sujetos al corte y el papel de los diferentes sectores que participan en el proceso de aprovechamiento. Algunos autores afirman que la cosecha de PFNM representa una opción viable de manejo forestal que puede ser congruente con la conservación de los bosques (Negi et al., 2011). En este artículo se evaluó esta noción usando como ejemplo el aprovechamiento de vara en los BTC de la cuenca del Pacífico en el occidente de México. Para ello, se partió de una revisión de estudios de caso, de la tendencia histórica del aprovechamiento de vara en esta región, así como de los resultados aportados por estudios referentes a la respuesta de las plantas al corte y a los efectos a nivel de comunidad de este aprovechamiento forestal. El trabajo finaliza con una serie de recomendaciones tendientes a modificar la forma en la que actualmente se maneja el recurso vara que permita asegurar la viabilidad de esta práctica en el largo plazo y su congruencia con la conservación de los BTC.

\section{Método}

Se llevó a cabo una revisión bibliográfica y de trabajos del sector gubernamental para el periodo 1987-2012 en torno al aprovechamiento de vara en los estados de Sonora, Sinaloa y Jalisco, donde el corte de vara se ha practicado con mayor intensidad. Con base en las notificaciones técnicas para el aprovechamiento de vara en la costa de Jalisco, proporcionadas por la Delegación Jalisco de la SEMARNAT, se hizo una recopilación de las cifras oficiales de extracción. Asimismo, se incorporó al análisis datos acerca de los niveles reales de extracción de vara y su efecto sobre la riqueza de especies, estructura y regeneración del bosque generados por el estudio de largo plazo iniciado en 1999 sobre el corte de vara en la costa de Jalisco, y que incluye entrevistas realizadas a los ejidatarios. Para dicho estudio se seleccionaron tres sitios de BTC con abundancia de vara blanca dentro del ejido Campo Acosta en el municipio de Tomatlán, Jalisco. De acuerdo con los testimonios de los ejidatarios, los tres sitios difieren en su historia de extracción: el Sitio 1 nunca tuvo corte, el Sitio 2, sólo tuvo un corte en el año 2003 y el Sitio 3 tuvo dos cortes; uno en 1985 y otro en 1998. Los sitios fueron cortados en cada ocasión por jornaleros en la forma convencional y con autorización oficial. Para el análisis de la composición y estructura de la vegetación y del 
rebrote, en cada sitio se establecieron tres parcelas cercadas de $60 \times 125 \mathrm{~m}\left(7,500 \mathrm{~m}^{2}\right)$ cada una. En cada parcela se realizó un censo de vegetación usando diez transectos rectangulares de $50 \times 2 \mathrm{~m}$, paralelos y separados entre sí por una distancia de $10 \mathrm{~m}$, sumando una superficie total censada de 0.1 ha. En cada transecto se midió la altura máxima y el diámetro de todos los tallos mayor o igual a $1 \mathrm{~cm}$ a $1.3 \mathrm{~m}$ de altura (DAP), además de registrar la identidad taxonómica de cada tallo. En este artículo se presentan resultados puntuales derivados de dicho estudio para ejemplificar con datos cuantitativos los niveles reales de extracción de vara al que se somete el BTC, la supervivencia de rebrotes y tocones, las consecuencias de la extracción en la capacidad de regeneración de las especies aprovechadas, así como el efecto de las intervenciones en la riqueza de especies y la estructura de los sitios. Además, para evaluar el crecimiento de los rebrotes, se diseñó un experimento de podas controladas de los rebrotes en tocones del Sitio 2. Para ello se seleccionó a un año del corte (2004) una muestra de 180 tocones con diferente densidad de rebrotes, los cuales se sometieron a tres tratamientos de poda (60 tocones por tratamiento): $\sin$ poda, poda del $50 \%$ y poda del $75 \%$ del total de los rebrotes presentes en el tocón, dejando intactos los rebrotes más vigorosos. La información completa de estos estudios, así como los detalles de los análisis estadísticos y el contexto general del proyecto pueden consultarse en Rendón-Carmona $(2002,2011)$ y Rendón-Carmona et al. (2009).

\section{Resultados}

Origen del corte de varas y especies aprovechadas. El corte de vara del BTC para uso hortícola en México se originó en Sonora a principios de 1960, con el aprovechamiento de Croton fantzianus F.Seym., una especie de vara blanca que forma densas poblaciones silvestres en el BTC de las partes bajas del municipio de Álamos, así como en los municipios de Quiriego, Navojoa y Rosario (Lindquist, 1999, 2000). En general, la Cuenca del Río Mayo a la que pertenecen estos municipios, se ha destacado por extraer vara, manteniéndola como una de las principales actividades forestales no maderables que genera ingresos económicos muy importantes para la población rural (Lindquist, 1999, 2000; Guevara, 2000; Palacios et al., 2009). En el estado de Sinaloa, el corte de vara se inició diez años después que en Sonora, abarcando prácticamente toda la entidad (12 de los 18 municipios: Choix, Concordia, Cosalá, Culiacán, El Fuerte, Elota, El Rosario, Mazatlán, Mocorito, Salvador Alvarado, San Ignacio y Sinaloa). La extracción se concentra en cinco especies del género Croton, conocidas también como vara blanca: C. alamosanus Rose, $C$. flavescens Greenm., C. reflexifolius Kunth, C. watsonii Standl. y C. septemnervius McVaugh; esta última es la especie de mayor extracción (López-Urquidez, 1997). En Jalisco, el aprovechamiento regulado de vara inició hasta 1996 en los municipios de Cabo Corrien- tes, Cuautitlán, La Huerta y Tomatlán. Según los documentos oficiales para el aprovechamiento de especies leñosas no maderables emitidos hasta el 2007 , en este periodo se autorizó el aprovechamiento de 22 especies y de un grupo indefinido de otras plantas leñosas en siete ejidos de esta región de Jalisco (Cuadro 1). Las plantas que se aprovechan pertenecen a 15 géneros y siete familias con cualidades para ser empleadas como varas. Destaca la familia Fabaceae que incorpora al mayor número de especies (diez especies; Cuadro 2). Aun cuando el grupo de especies aprovechadas es diverso, tanto en Jalisco como en Sinaloa, la mayor proporción de las varas cortadas pertenecen al género Croton y por ejemplo, en el ejido Campo Acosta en Jalisco, el 76\% pertenecen a $C$. septemnervius.

Uso de las varas del BTC. Las varas se usan principalmente como tutores en el cultivo de hortalizas y algunas frutas. Por ejemplo, las varas de $C$. fantzianus se usan en los cultivos de jitomate y uva en los extensos campos agrícolas de Baja California, Sinaloa y Sonora (Lindquist, 1999, 2000; Guevara, 2000; Palacios et al., 2009), aunque también se usan ampliamente en la construcción de paredes y techos de casas en las comunidades indígenas del sur de Sonora (Yetman et al., 2000). Este tipo de construcción prehispánica es común en todo México y sin duda diferentes especies de Croton, por su resistencia a hongos y termitas, fueron utilizadas tradicionalmente en la construcción de casas con paredes de lodo ("wattle and daub", ramas entrelazadas y lodo). Al igual que en Sonora, en Sinaloa y Jalisco, el grueso de la cosecha actual de vara blanca se dedica a la producción de tutores para las zonas hortícolas de jitomate, berenjena, calabacita, chile y pepino (López-Urquidez, 1997). En Jalisco, la extracción de vara blanca se realizaba originalmente para abastecer las plantaciones hortícolas del municipio de Sayula; sin embargo, entrevistas realizadas a jornaleros cortadores en el año 2000 (Rendón-Carmona, 2002), indican que parte del material extraído era transportado hasta campos agrícolas de Sinaloa. Actualmente, en el ejido Campo Acosta, el uso de tutores (principalmente en el cultivo de chile y con menor intensidad de pepino) atiende principalmente la demanda local; un fenómeno creciente que modera la salida a otros mercados y privilegia el consumo local.

Tallas de los árboles sujetos al corte. En el BTC del sur de Sonora, en los sitios con abundancia de Croton fantzianus, el diámetro de los tallos cortados es muy variable, pero las varas de mayor demanda y extracción varían entre 2.5 y 3.8 $\mathrm{cm}$ de diámetro (Lindquist, 2000). Las plantas de Croton septemnervius, que preferencialmente se cortan en la costa de Jalisco, son individuos adultos monopódicos del que se obtiene una sola vara. Aunque la regulación forestal fija un diámetro de corte de $3.8 \mathrm{~cm}$, el grosor de los tocones en áreas aprovechadas por primera vez (y por lo tanto de los tallos cortados) varía de 2 y hasta $9 \mathrm{~cm}$, y es de 4 a $6 \mathrm{~cm}$ el 
Humberto Rendón-CARMONa ET AL.

Cuadro 1. Permisos para corte de vara otorgados durante el periodo 1996-2007 a ejidos de la costa de Jalisco, México. Fuentes de información: notificaciones oficiales de la SEMARNAT (Delegación Jalisco). N.E. = no especificado.

\begin{tabular}{|c|c|c|c|c|c|c|c|c|}
\hline Ejido & Municipio & $\begin{array}{l}\text { Superficie } \\
\text { autorizada } \\
\text { (ha) }\end{array}$ & $\begin{array}{l}\text { Año del } \\
\text { permiso }\end{array}$ & $\begin{array}{c}\text { Periodo } \\
\text { autorizado }\end{array}$ & Especies autorizadas & $\begin{array}{l}\text { Volumen } \\
\text { autorizado } \\
\left(\mathbf{m}^{3}\right)\end{array}$ & $\begin{array}{l}\text { No. varas } \\
\text { cortadas en } \\
\text { el periodo }^{1}\end{array}$ & Fuente \\
\hline $\begin{array}{l}\text { Ejido Plan } \\
\text { de Méndez }\end{array}$ & Cuautitlán & 115 & 1996 & $\begin{array}{c}3 \text { años } \\
(1996-1999)\end{array}$ & $\begin{array}{l}\text { Otate } \\
\text { (Arthrostylidium } \\
\text { longiflorum) }\end{array}$ & $1,729.5$ & 330,688 & Hueso, 1996 \\
\hline $\begin{array}{l}\text { P. P. } \\
\text { Tiradores }\end{array}$ & La Huerta & 214 & 1997 & $\begin{array}{c}3 \text { años } \\
(1997-2000)\end{array}$ & $\begin{array}{l}\text { Pata de venado } \\
\text { (Bauhinia latifolia), } \\
\text { Iguanero (Caesalpinia } \\
\text { eriostachys), Camotillo, } \\
\text { Jediondillo, Guajillo, } \\
\text { Tepezapote, Cacavanance, } \\
\text { Varablanca, etc. }\end{array}$ & 739.9 & 141,472 & Lomelí, 1996 \\
\hline $\begin{array}{l}\text { Comunidad } \\
\text { indígena de } \\
\text { Tomatlán }\end{array}$ & Tomatlán & 185 & 1997 & N.E. & $\begin{array}{l}\text { Canelilla y otras } \\
\text { comunes }\end{array}$ & 400.0 & 76,482 & Ortega, 1997 \\
\hline $\begin{array}{l}\text { Ejido Lázaro } \\
\text { Cardenas }\end{array}$ & Tomatlán & 840 & 1999 & $\begin{array}{c}2 \text { años } \\
(1999-2001)\end{array}$ & $\begin{array}{l}\text { Canelilla (Croton sp.), } \\
\text { Cuatalaca, entre otras }\end{array}$ & $1,426.0$ & 272,658 & Barrios, 1999 \\
\hline Morelos & La Huerta & $1,522.5$ & 2006 & $\begin{array}{c}5 \text { años } \\
(2006-2011)\end{array}$ & $\begin{array}{l}\text { Iguanero, Garrapato, } \\
\text { Parotilla, Cacahuananche, } \\
\text { Coral, Guayabillo, } \\
\text { Tepemezquite, Paracata, } \\
\text { Barcino, Tlahuitole, Palo } \\
\text { amarillo, Vara de botoncillo, } \\
\text { Vara blanca, Cuero de venado, } \\
\text { Espino blanco, Otras. }\end{array}$ & 278.6 & 53,270 & $\begin{array}{l}\text { Delegación Estatal } \\
\text { SEMARNAT Jalisco }\end{array}$ \\
\hline Hidalgo & $\begin{array}{l}\text { La Huerta o } \\
\text { Cuautitlán }\end{array}$ & N.E. & 2007 & N.E. & $\begin{array}{l}\text { Incorporación de no } \\
\text { maderables (varas) }\end{array}$ & $2,386.2$ & 456,254 & \\
\hline $\begin{array}{l}\text { NCPA Adolfo } \\
\text { López Mateos }\end{array}$ & $\begin{array}{c}\text { Cabo } \\
\text { Corrientes }\end{array}$ & N.E. & 2007 & N.E. & $\begin{array}{l}\text { Aprovechamiento de } \\
\text { no maderables }\end{array}$ & 1,035 & 197,897 & \\
\hline
\end{tabular}

'Valor de extracción estimado en el presente estudio, considerando según Barrios (1999) que 0.523 m³ rollo equivalen a 100 varas (ver texto).

diámetro de corte más frecuente (Figura 1A). La altura de corte de los tallos también es muy variable, desde el nivel del suelo hasta una altura máxima de $1.25 \mathrm{~m}$, pero la mayoría de los tallos son cortados a una altura entre 11 y $20 \mathrm{~cm}$ del suelo (Figura 1B).

Sectores que participan en el corte y comercialización de las varas. De acuerdo con Lindquist (2000), además de los propietarios de la tierra, en Sonora existen tres sectores que participan en la extracción de la vara: cortadores, contratistas e intermediarios. Los cortadores son jornaleros contratados $e x$ profeso que cosechan hasta 200 tallos/día en la temporada de corte (en cualquier época del año o concentrada en los meses de sequía), aunque en algunos casos los cortadores pueden ser los propios ejidatarios. El segundo sector es el de los contratistas, que pagan una comisión a los dueños de los terrenos de $\$ 200$ a $\$ 400$ pesos por cada millar de varas cortadas por los jornaleros a su cargo. Los contratis- tas en ocasiones también compran las varas en los ranchos (\$0.60/vara) y en los ejidos (\$1.00/vara). En el tercer sector están los intermediarios que se encargan de transportar y de comercializar la vara desde los sitios de corte hasta los campos agrícolas de Caborca, Ciudad Obregón, Guaymas y Hermosillo. Las varas también se transportan hasta los campos agrícolas de Sinaloa (en Culiacán y Guasave) y de Baja California (en Camalú, Ensenada y San Quintín,). El costo que adquieren las varas varía según el lugar donde se comercialicen. Por ejemplo, en el año 2000 los horticultores en Sonora compraban la vara entre $\$ 2.40$ y $\$ 2.50$ pesos, en Sinaloa entre $\$ 1.80$ y $\$ 2.00$ y en Baja California entre $\$ 3.30$ y $\$ 3.50$. El mejor mercado para la venta de varas está en los campos agrícolas de este último estado y los grandes intermediarios lo prefieren a pesar de que los gastos de traslado sean más altos (un camión que típicamente transporta 12,000 varas hacia Baja California tiene un costo de $\$ 7,000$ pesos en gasolina, peajes y gastos del chofer). 

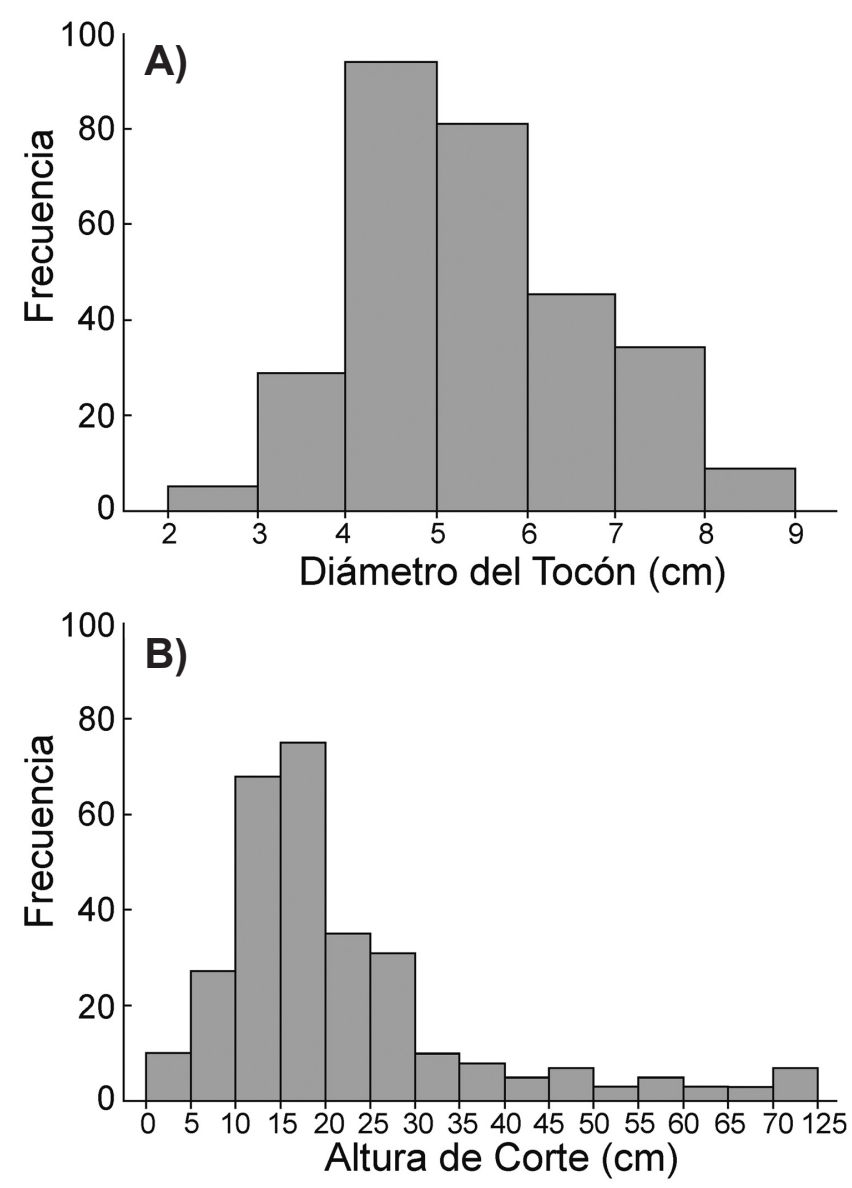

Figura 1. Distribución de tamaños de los tocones: A) diámetro basal del tocón $(\mathrm{cm})$ y, B) altura de corte $(\mathrm{cm})$ de las nueve especies de vara aprovechadas en el bosque tropical caducifolio en el ejido Campo Acosta, municipio de Tomatlán, Jalisco, México. Área total de muestreo: 0.3 ha. $\mathrm{N}=297$ tocones.

En Jalisco, al igual que en Sonora, tres sectores participan en la extracción; sin embargo, difiere en que todo el aprovechamiento tiene lugar en áreas ejidales, y prácticamente hasta el año 2000, los cortadores provenían de Sinaloa. La única participación de los ejidatarios consistía en rentar sus terrenos por un periodo de 2 a 3 años y recibir $\$ 2.00$ por un bulto de varas formado de 3 a 5 piezas cada uno. En los ejidos de la costa de Jalisco, los jornaleros cortaban en promedio 221 varas/día por lo cual recibían un pago de \$195.50/día (Rendón-Carmona, 2002). El papel del intermediario es transportar las varas desde los centros de acopio en los ejidos a los campos hortícolas en Jalisco o hasta Sinaloa. Por la venta de un cargamento completo de vara (2,000 bultos, entre 6 y 10 mil varas) destinado a los campos sinaloenses, el intermediario obtiene $\$ 34,000$ pesos ( $\$ 17.00$ por bulto). De esta cantidad el intermediario tiene que pagar $\$ 8,000$ pesos por concepto de flete, $\$ 7,077$ por los jornales de los cortadores (36.2 jornales a $\$ 195.50$ diarios), y $\$ 4,000$ por la compra de las varas a los ejidatarios $(8,000$ varas a $\$ 0.50$ ), por lo que la ganancia neta del intermediario es de aproximadamente $\$ 15,000$ pesos por viaje (RendónCarmona, 2002).

Respuesta de las plantas al corte y efectos de la extracción a nivel de la comunidad vegetal. La mayoría de las especies de vara aprovechadas en Jalisco son plantas nativas del BTC con capacidad de responder al corte produciendo numerosos rebrotes. A excepción del barcino (Cordia elaeagnoides DC), que produce en promedio seis rebrotes/tocón, el resto de las especies producen entre 11 y 22 rebrotes/tocón (Cuadro 3). El crecimiento de los rebrotes difiere significativamente entre las especies aprovechadas, con una variación en diámetro basal y altura máxima de 3 a $5 \mathrm{~mm}$ y 29 a $84 \mathrm{~cm}$, respectivamente (Cuadro 3). En el caso de Croton septemnervius, la especie de mayor extracción, la capacidad de rebrotar es muy alta, ya que puede producir hasta 53 rebrotes
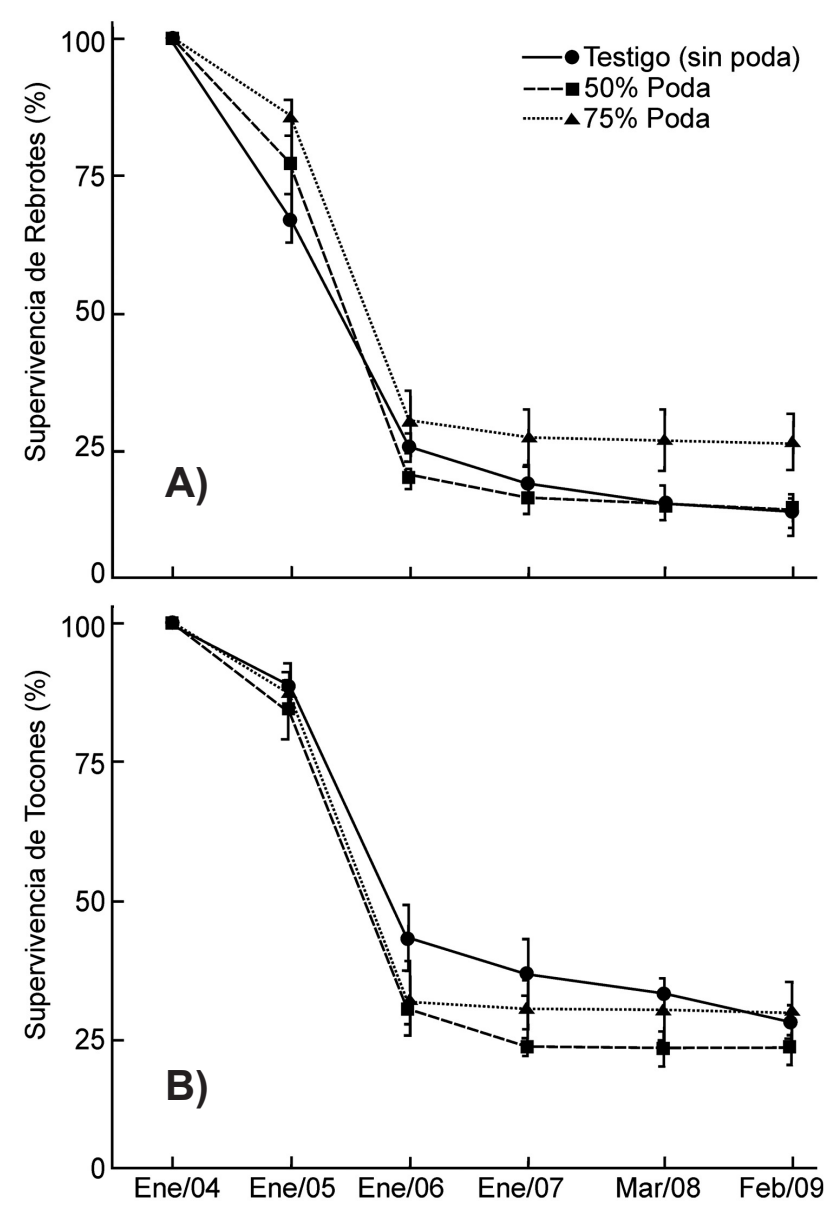

Figura 2. Porcentaje de supervivencia de rebrotes (A) y de tocones (B) por nivel de poda en Croton septemnervius en el periodo 20042009 en el ejido Campo Acosta, municipio de Tomatlán, Jalisco, México. $\mathrm{N}=60$ tocones por tratamiento de poda (sin poda, poda al $50 \%$ y poda al $75 \%$ del total de los rebrotes en el tocón). Los valores representan el promedio de tres sitios (promedio de 20 tocones por sitio), y las líneas verticales en cada punto el error estándar. 
Cuadro 2. Especies del bosque tropical caducifolio con evidencia de corte de vara en el Ejido Campo Acosta, Jalisco, México, en dos sitios que difieren en la historia de extracción (con uno y dos cortes). AOC = con autorización oficial para corte, A = árbol, Ar = Arbusto.

\begin{tabular}{|c|c|c|c|c|c|}
\hline & Nombre común & Especie & Familia & $\begin{array}{l}\text { Forma de } \\
\text { crecimiento }\end{array}$ & $\mathrm{AOC}$ \\
\hline 1 & Tabebuia & Tabebuia impetiginosa (Mart. ex DC.) Standl. & Bignoniaceae & A & No \\
\hline 2 & Barcino & Cordia elaeagnoides DC. & Boraginaceae & A & $\mathrm{Si}$ \\
\hline 3 & Chinito & Aeschynomene amorphoides (S.Wats.) Rose ex B.L.Rob. & Fabaceae & $\mathrm{Ar}$ & No \\
\hline 4 & Tlahuitole, Llorasangre & Apoplanesia paniculata C. Presl & Fabaceae & A & $\mathrm{Si}$ \\
\hline 5 & Pata de venado & Bauhinia ungulata L. & Fabaceae & $\mathrm{Ar}$ & $\mathrm{Si}$ \\
\hline 6 & - & Brongniartia pacifica McVaugh & Fabaceae & $\mathrm{Ar}$ & No \\
\hline 7 & Iguanero & Caesalpinia eriostachys Benth. & Fabaceae & A & $\mathrm{Si}$ \\
\hline 8 & Coral, Frijolillo & Caesalpinia platyloba S.Wats. & Fabaceae & $\mathrm{A}, \mathrm{Ar}$ & $\mathrm{Si}$ \\
\hline 9 & Catizpilla & Gliricidia sepium (Jacq.) Kunth ex Walp. & Fabaceae & $\mathrm{A}, \mathrm{Ar}$ & $\mathrm{Si}$ \\
\hline 10 & Guajillo & Leucaena lanceolata S.Watson & Fabaceae & A & Si \\
\hline 11 & Botoncillo, Cuero de indio & Lonchocarpus sp. & Fabaceae & A & No \\
\hline 12 & Tepemezquite & Lysiloma divaricatum (Jacq.) J.F.Macbr. & Fabaceae & A & $\mathrm{Si}$ \\
\hline 13 & Chamizo & Casearia corymbosa Kunth & Salicaceae & $\mathrm{A}, \mathrm{Ar}$ & No \\
\hline 14 & Trementina & Casearia dolichophylla Standl. & Salicaceae & A & No \\
\hline 15 & Ocotillo & Casearia tremula (Griseb.) Griseb. ex C.Wright & Salicaceae & A & $\mathrm{Si}$ \\
\hline 16 & Canelilla & Croton septemnervius McVaugh & Euphorbiaceae & $\mathrm{A}, \mathrm{Ar}$ & $\mathrm{Si}$ \\
\hline 17 & Guayabillo & Piranhea mexicana (Standl.) Radcl.-Sm & Euphorbiaceae & A & No \\
\hline 18 & - & Ruprechtia fusca Fernald & Polygonaceae & A & No \\
\hline 19 & Crucecilla & Randia sp. & Rubiaceae & $\mathrm{Ar}$ & No \\
\hline
\end{tabular}

por tocón. Bajo un esquema de manejo del rebrote (es decir, aplicando podas controladas de los rebrotes menos vigorosos del tocón) la supervivencia de los rebrotes se incrementa notablemente (Figura 2A). Por ejemplo, cuando se remueven tres cuartos de los rebrotes del tocón, la supervivencia de los rebrotes restantes después de cinco años es del $26 \%$, mientras que cuando no se aplica ninguna poda o se poda sólo la mitad de los rebrotes, la supervivencia se reduce al $14 \%$ (una diferencia entre tratamientos del 54\%). Sin em- bargo, aún con la poda selectiva e intensa de los rebrotes, la supervivencia es muy limitada, ya que en el mejor de los casos, únicamente una cuarta parte sobrevive (Figura 2A). La supervivencia de los tocones, aunque no se relaciona con la densidad de rebrotes o con la intensidad de poda, también es muy baja, pues es cercana al $30 \%$ cinco años después de la cosecha (Figura 2B). A nivel de la composición florística de la comunidad, los datos sugieren que el número de cortes de vara en los sitios está inversamente relacionado con la

Cuadro 3. Número de rebrotes por tocón, diámetro basal (DB) y altura de los rebrotes de siete especies leñosas del bosque tropical caducifolio proveedoras de vara en el ejido Campo Acosta, municipio de Tomatlán, Jalisco, México. Valores promedio (Prom.) \pm 1 Error Estándar (E.E.) a un año del corte de vara (enero del 2004). Letras diferentes en la misma columna indican diferencias significativas entre las especies (SNK posterior a un ANOVA; $\mathrm{P}<0.05)$.

\begin{tabular}{|c|c|c|c|c|c|c|c|}
\hline \multirow[t]{2}{*}{ Especie } & \multirow{2}{*}{$\begin{array}{c}\text { Número } \\
\text { de tocones* }\end{array}$} & \multicolumn{2}{|c|}{ Número de rebrotes } & \multicolumn{2}{|c|}{ DB rebrotes $(\mathrm{mm})$} & \multicolumn{2}{|c|}{ Altura rebrotes $(\mathrm{cm})$} \\
\hline & & Prom. & E.E. & Prom. & E.E. & Prom. & E.E. \\
\hline Croton septemnervius & 181 & $15.1^{\mathrm{ab}}$ & 0.6 & $2.8^{\mathrm{c}}$ & 0.1 & $35.7^{\mathrm{bc}}$ & 1.3 \\
\hline Lonchocarpus sp. & 41 & $10.7^{\mathrm{ab}}$ & 0.6 & $5.3^{\mathrm{b}}$ & 0.3 & $64.4^{\mathrm{ab}}$ & 4.5 \\
\hline Randia sp. & 9 & $14.4^{\mathrm{ab}}$ & 3.4 & $3.2^{\mathrm{bc}}$ & 0.3 & $29.4^{c}$ & 3.9 \\
\hline Piranhea mexicana & 7 & $21.6^{\mathrm{a}}$ & 5.2 & $3.8^{\mathrm{bc}}$ & 0.4 & $37.0^{\mathrm{bc}}$ & 5.8 \\
\hline Cordia elaeagnoides & 4 & $6.0^{\mathrm{b}}$ & 0.9 & $7.6^{\mathrm{a}}$ & 1.1 & $84.3^{\mathrm{a}}$ & 17.6 \\
\hline Caesalpinia eriostachys & 3 & $15.0^{\mathrm{ab}}$ & 1.5 & $2.7^{\mathrm{c}}$ & 0.5 & $35.7^{\mathrm{bc}}$ & 7.2 \\
\hline \multirow[t]{3}{*}{ Ruprechtia fusca } & 3 & $11.7^{\mathrm{ab}}$ & 2.4 & $3.6^{\mathrm{bc}}$ & 0.6 & $39.3^{\mathrm{bc}}$ & 8.7 \\
\hline & & \multicolumn{2}{|c|}{$F=3.4815$} & \multicolumn{2}{|c|}{$F=29.804$} & \multicolumn{2}{|c|}{$F=15.146$} \\
\hline & & \multicolumn{2}{|c|}{$P=0.0026$} & \multicolumn{2}{|c|}{$P=0.0001$} & \multicolumn{2}{|c|}{$P=0.0001$} \\
\hline
\end{tabular}

*Número total de tocones por especie. Tocones registrados en 30 transectos rectangulares de 50 × 2 m (10 transectos en cada una de las tres parcelas de 7,500 $\mathrm{m}^{2}$ distribuidas aleatoriamente dentro del sitio). El corte de vara se realizó en enero del 2003 por los jornaleros en áreas autorizadas para corte dentro del ejido Campo Acosta. 


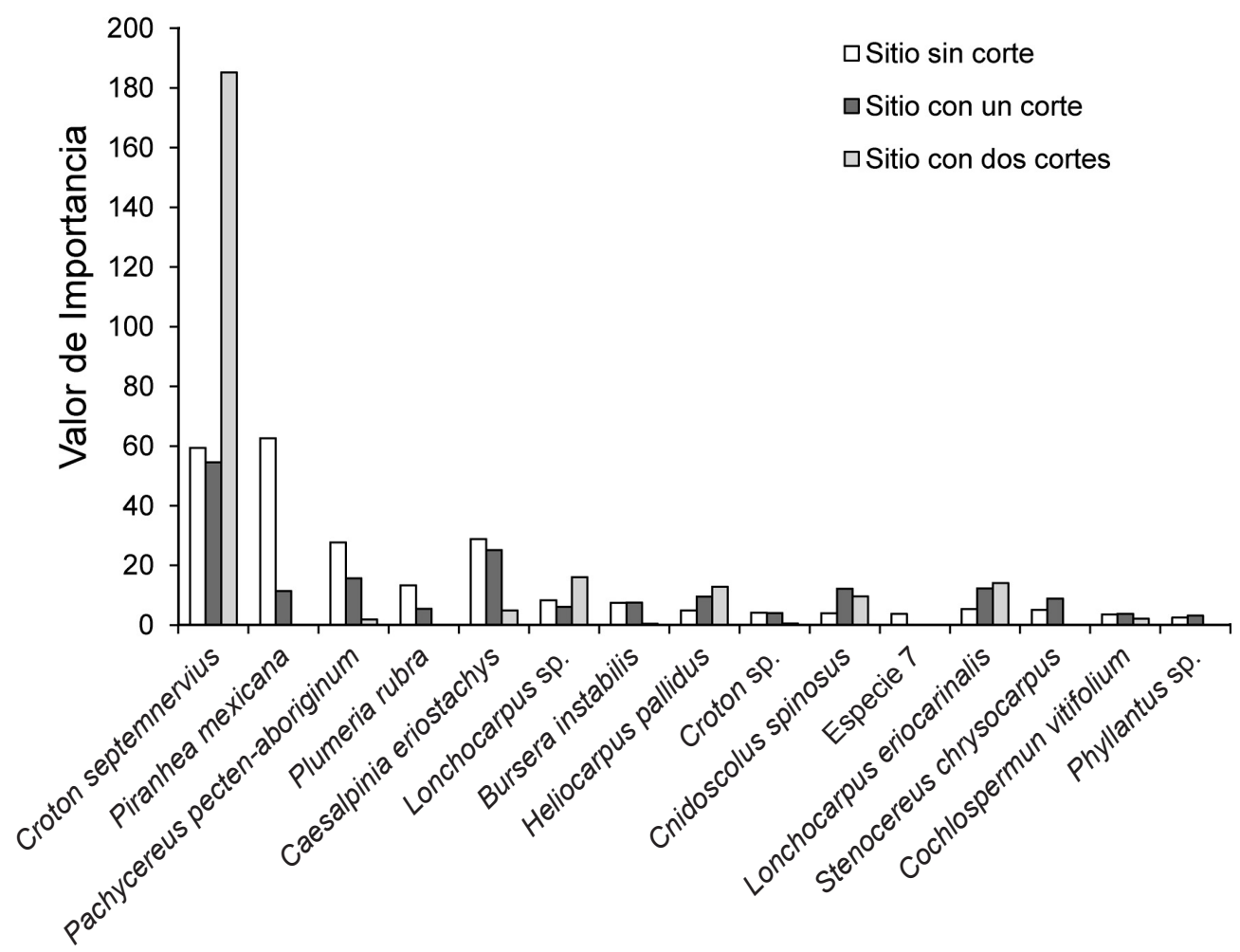

Figura 3. Valor de Importancia de las 15 especies leñosas con mayor densidad en tres áreas de bosque tropical caducifolio con diferente historia de corte de vara (sin corte, con un corte, con dos cortes) en el ejido Campo Acosta, municipio de Tomatlán, Jalisco, México. En

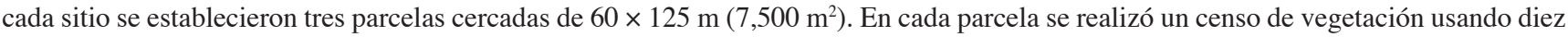
transectos rectangulares de $50 \times 2 \mathrm{~m}$.

riqueza de especies, ya que sitios sin corte, con uno y con dos cortes, presentan 65,50 y 38 especies leñosas, respectivamente (individuos con DAP $\geq 1.0 \mathrm{~cm}$ ), y que el aumento en los eventos de corte genera cambios en los patrones de dominancia de las especies en los sitios aprovechados. Dichos cambios se aprecian en una disminución en la densidad de algunas especies y en la desaparición de otras, así como en una fuerte dominancia de Croton septemnervius en densidad, área basal y número de tallos, y por lo tanto en la jerarquía de valor de importancia relativo de las especies en la comunidad (Figura 3).

\section{Discusión}

La presente investigación muestra que el aprovechamiento de tutores del BTC se realiza principalmente en dos especies de vara blanca, Croton fantzianus y C. septemnervius, pero que dada la alta demanda de tutores para la horticultura en México, un diverso grupo de otras especies leñosas del BTC, principalmente leguminosas, también se aprovechan aunque con menor intensidad. A pesar de que el corte de vara se inició oficialmente hace 17 años, cuando se otorgaron las primeras autorizaciones en los ejidos de la costa de Jalisco se encontró que la extracción inició hace casi 40 años (mediados de la década de 1970), manteniéndose desde entonces como una actividad forestal importante (RendónCarmona, 2002). En el caso de Sonora, Lindquist (2000) menciona que aunque existe una larga tradición en el corte de vara con diferentes propósitos (especialmente para construcción de casas, techos y cercos), el aprovechamiento de vara blanca con fines comerciales comenzó en la década de 1960. El hecho de que por tanto tiempo se haya practicado esta actividad forestal en ausencia de un marco de regulación oficial tiene serias implicaciones para la conservación del BTC en esta región del país. Por un lado, existe una fuerte presión sobre las poblaciones de las especies nativas de Croton, y por el otro, se desconoce la magnitud del impacto de la extracción sobre éstas y otras especies leñosas que se usan como sustitutos de la vara. En consecuencia, es probable que la sostenibilidad de la cosecha de muchas de estas especies de las que parcialmente depende la gente está en riesgo, por lo que es urgente realizar investigación que busque maximizar el uso productivo de estos recursos, a la vez que se minimice la probabilidad de extinción como una alternativa económica (Weinbaum et al., 2013).

De los tres sectores involucrados en la extracción del recurso vara (cortadores, contratistas e intermediarios), más los propietarios de las áreas aprovechadas, resulta evidente 
que estos últimos se encuentran en desventaja económica con respecto a los intermediarios. De acuerdo con RendónCarmona (2002), los intermediarios obtienen una utilidad del $47 \%$ de la venta de la vara, mientras que la de los propietarios es de apenas el 12\%. Esta disparidad obedece en parte a que los ejidatarios desconocen el costo que las varas alcanzan en el mercado final, pero también a que carecen de los contactos y los medios de transporte y derivan un beneficio económico cuya factura, en ausencia de un plan de manejo, se carga directamente al ecosistema. Para la parte baja de la Cuenca del Río Mayo en Sonora, Palacios et al. (2009) calcularon que la explotación de la vara blanca es la segunda actividad forestal-después de la recolección de leña-, que más beneficios económicos genera, con un valor aproximado de $\$ 21,964$ pesos ha $^{-1} \mathrm{año}^{-1}$, aunque sólo una fracción de este valor queda en manos de los propietarios. Esta situación es semejante en la región de Álamos, Sonora, donde el ingreso en cada sector que participa en la comercialización de la vara aumenta significativamente; sin embargo, el ingreso más bajo es el de los ejidatarios que dependen parcial o totalmente de este ingreso para cubrir sus necesidades básicas de subsistencia (Lindquist, 2000).

Aunque la recolecta se ha realizado durante al menos cinco décadas, existen muy pocos estudios cuantitativos que documenten la dinámica de la regeneración de las especies de vara blanca, y menos aún para el resto de las especies leñosas aprovechadas. En algunos estudios, la edad de los rebrotes se infiere a partir de las observaciones de los cortadores y los intermediarios. Por ejemplo, en la región de Álamos, Sonora, la percepción de los cortadores de vara es que el bosque se renueva mediante procesos naturales y que en un periodo de 5 a 7 años los rebrotes de Croton fantzianus alcanzan la talla comercial (Lindquist, 1999). En Jalisco, los ejidatarios familiarizados con el corte de vara tienen una percepción semejante a los jornaleros de Sonora en el sentido de que los árboles cortados se recuperan en pocos años (3-5). Sin embargo, contrario a estas percepciones, se encontró para $C$. septemnervius, que aun disminuyendo la densidad de los rebrotes por tocón, los rebrotes remanentes podrían alcanzar la talla comercial a los 13 años si se practica una poda del 75\%, o a los 18 años cuando se poda el $50 \%$ o no se realiza ninguna poda; una notable diferencia $(>50 \%)$ entre la precepción de los productores y el crecimiento real. Otro atributo de la regeneración vegetativa de la vara blanca que no es percibido por los productores es que la supervivencia de los rebrotes y tocones es baja, aunque puede aumentar si se aplican podas. Es decir, contrario a la percepción manifestada por los productores, este trabajo muestra que la regeneración natural de la vara blanca presenta serias restricciones para la recuperación exitosa de sus poblaciones y la provisión de este recurso en el largo plazo. Para determinar con mayor precisión el componente ecológico de la sostenibilidad de este aprovechamiento forestal son necesarios nuevos estudios que incluyan más sitios, mayores intervalos temporales, y un análisis demográfico más detallado de las especies más utilizadas. Un enfoque experimental del corte de vara en estos estudios es también fundamental para probar el éxito de distintos niveles de extracción.

A excepción de Campo Acosta en Jalisco, no existen tampoco estudios que hayan cuantificado el efecto de la extracción de vara en la composición florística y estructura de la comunidad de los sitios donde ocurre la extracción. En este ejido, se encontraron cambios en los patrones de dominancia-diversidad que originan comunidades con menos riqueza de especies y con dominancia creciente de Croton septemnervius a medida que aumenta el número de cortes en los sitios (Rendón-Carmona et al., 2009). Desde el punto de vista de la disponibilidad de tallos que proveen de tutores para la horticultura, el aumento en la dominancia de esta especie es positivo para los jornaleros y dueños de las tierras, ya que el potencial de extracción es mayor. Sin embargo, dicha dominancia ocurre a expensas de una reducción en la riqueza y abundancia de especies arbóreas del bosque primario, mientras que otras especies de carácter secundario aumentan su densidad (Rendón-Carmona et al., 2009). A este respecto, Lindquist (2000) reporta que en Sonora, sitios de BTC con historia de corte de vara se encuentran severamente alterados y que debido al uso intensivo su permanencia futura no está garantizada.

La regulación técnica para el aprovechamiento de PFNM, en México, se encuentra en la Norma Oficial Mexicana NOM-005-RECNAT-1997, actualmente Proyecto de Norma Oficial Mexicana PROY-NOM-005-SEMARNAT2012 (Diario Oficial de la Federación 30 de julio 2012). En esta norma se establecen los criterios y especificaciones para realizar el aprovechamiento sustentable de los PFNM existentes en los ecosistemas forestales; bosques de clima templado, frío, selvas y zonas áridas y semiáridas. No obstante esta normatividad, las notificaciones son deficientes, por lo que la extracción de vara se basa en una regulación poco clara que fomenta la extracción ilegal. Por ejemplo, en algunos de los expedientes técnicos de Jalisco únicamente se indican los nombres comunes de las especies autorizadas para corte o son agrupadas bajo la denominación de "otras". Sólo por citar un caso, las especies autorizadas para el corte en el municipio de Tomatlán se especifican en los permisos como "Canelilla y otras comunes tropicales", o como "Canelilla (Croton sp.), Cuatalaca, entre otras" (ver Cuadro 1). Esta ambigüedad en la nomenclatura ha conducido a la cosecha indiscriminada de plantas usadas como tutores. En el caso particular de Jalisco, las notificaciones oficiales autorizan el aprovechamiento de 22 especies leñosas del BTC; sin embargo, se extraen varas de 19 especies y de éstas, únicamente diez cuentan con autorización oficial (ver Cuadro 2). Por lo tanto, el corte de vara en esta región incluye a nueve especies del BTC que se extraen de forma ilegal, mientras que se otorgan permisos para tres especies que ni siquiera se 
cortan. Esto confirma que la regulación técnica de aprovechamiento de vara en esta región del país es deficiente. De acuerdo con CONABIO (2009), éste es un problema generalizado en la gestión de los PFNM de México.

Otro problema relacionado con los permisos de corte de vara es que no indican para cada especie el volumen de extracción y únicamente mencionan que es necesario dejar en pie el $20 \%$ de los individuos maduros y a los individuos jóvenes. Sin embargo, estos lineamientos claramente no aplican por igual a todas las especies aprovechadas ya que la tolerancia al corte difiere entre las especies, y no es de esperar que la extracción del $80 \%$ de los individuos garantice que con el 20\% restante se puedan mantener las poblaciones (Olmsted y Alvarez-Buylla, 1995; Ticktin, 2004). Además, las notificaciones técnicas parten de la base de que las especies sujetas al corte tienen la capacidad de regenerarse en forma sexual (por semillas) y asexual (por rebrotes) y que estas medidas las llevarán a una "regeneración satisfactoria". Sin embargo, este criterio no toma en cuenta que dicha capacidad varía entre las especies y que depende no sólo de la producción de semillas, de la tasa de reclutamiento de plántulas, de la tasa de crecimiento y la supervivencia de los rebrotes, sino también de su interacción con las condiciones climáticas o la disponibilidad de recursos (LópezToledo et al., 2012), que son altamente variables en el BTC. $\mathrm{Al}$ respecto, los estudios en la región de Chamela muestran que del 2004 al 2009 la mortalidad de rebrotes y de tocones de Croton septemnervius fue muy alta (> 70\%). En dicho periodo se presentaron dos años consecutivos de muy baja precipitación anual (c. $300 \mathrm{~mm}$ ), equivalentes al 38\% del valor promedio anual para la región, lo cual posiblemente haya afectado la resiliencia o la habilidad de las plantas para recuperarse del corte (López-Toledo et al., 2012). Esto resalta la importancia de evaluar la respuesta de las plantas sometidas a cosecha en una perspectiva de largo plazo que permita incorporar el impacto de la variabilidad climática en los criterios de aprovechamiento forestal.

Un elemento explícito en los permisos de aprovechamiento de vara es el volumen total de extracción en los sitios autorizados para corte. Sin embargo, observamos que el número total de tallos cortados no coincide con el total estimado a partir de las cifras autorizadas. Por ejemplo, si consideramos que $0.523 \mathrm{~m}^{3}$ de madera en rollo equivalen a 100 varas (Barrios, 1999: Notificación oficial de aprovechamiento de recursos forestales con fines comerciales para el municipio de Tomatlán) y que el volumen total autorizado de 1996 a 2007 en los ejidos de la costa de Jalisco fue de $4,574 \mathrm{~m}^{3}$ (únicamente en los ejidos en los que se especifica la superficie autorizada; ver Cuadro 1), la extracción total esperada es de 874,570 varas en 2,877 ha; un promedio de 304 varas ha $^{-1}$. En contraste con esta estimación, la extracción real de vara registrada en los ejidos de esta región varia de 990 a 1,910 varas ha $^{-1}$; una diferencia de tres a seis veces mayor al número total de varas oficialmente autori- zadas para el mismo periodo y superficie. A este respecto, Lindquist (2000) reporta que aunque se requieren permisos para el corte en lugares y en cantidades especificados por la autoridad, raramente existe vigilancia para que esta regulación se cumpla, lo que ha conducido a una disminución en las densidades de vara blanca por hectárea en el BTC del sur de Sonora.

Aunado a la problemática general de gestión de los PFNM en México, los permisos para corte de vara no toman en cuenta aspectos ecológicos fundamentales en los criterios de aprovechamiento, como tiempos de descanso (rotación de las áreas aprovechadas) y el disturbio ocasionado en el bosque por la apertura de brechas para el transporte de la vara (que implica el derribo de otros árboles, la creación de claros y la fragmentación del dosel). Estudios realizados en Sonora y Sinaloa señalan que debido al poco tiempo de descanso entre cortes, en los últimos años es más difícil encontrar tallos de tamaño apropiado para corte (Muro, 1987; Reyes-Olivas, 1993; Lemus-Vázquez y González-Guiza, 1995; López-Urquidez, 1997). Como un indicador de la creciente escasez de vara en Sonora por la sobreexplotación, para 1999 los horticultores ya adquirían tutores de baja calidad (delgados o deformes), condición que en años anteriores no era aceptada (Lindquist, 1999). Por otro lado, las medidas de mitigación en los expedientes para el corte de vara, aunque muy generales, no son tomadas en cuenta. Por ejemplo, bajo la suposición de que las áreas aprovechadas son extensas y por lo tanto existen corredores alternos que pueden servir de refugio a la fauna silvestre, las notificaciones indican que "la afectación hacia la fauna será nula o mínima" y que podrá conseguirse "dejando en pie todos los árboles con nidos o madrigueras, además de prohibirse la cacería". Sin embargo, se ha documentado que se corta más del $80 \%$ de los individuos maduros dejando en pie únicamente a los individuos con tallos deformes (Rendón-Carmona et al., 2009) y sin consideración sobre la presencia de madrigueras o nidos. Una situación similar se reporta para los estados de Sonora y Sinaloa (López-Urquidez, 1997; Lindquist, 2000).

Recomendaciones dirigidas hacia un aprovechamiento sostenible de la vara. La presente investigación sugiere que las prácticas actuales de aprovechamiento de vara para uso hortícola no son viables en el largo plazo, ni consistentes con el mantenimiento de la diversidad del bosque tropical caducifolio. Se considera que para alcanzar el objetivo de aprovechamiento sostenible de vara deberían implementarse las siguientes medidas: (1) evaluar la cobertura y la condición de los bosques en las áreas de BTC donde se autoriza el corte de vara, antes y después del aprovechamiento; (2) conocer, por medio de inventarios forestales, la distribución y abundancia de todas las especies del BTC que se aprovechan como tutores, así como los niveles de cosecha a los cuales están sometidas; (3) documentar la capacidad de regeneración por 
rebrote y por semilla de todas las especies sujetas a aprovechamiento y el aporte que tienen ambos mecanismos para la recuperación de las áreas sometidas a cosecha (de Freitas y Pinard, 2008; Busby et al., 2010; Levesque et al., 2011); (4) estudiar el efecto de la cosecha repetida en la supervivencia de las plantas y otros parámetros demográficos de las especies aprovechadas (Olmsted y Alvarez-Buylla, 1995; Anten et al., 2003; Marshall y Newton, 2003); y (5) analizar los probables cambios que el corte selectivo pueda estar generando a nivel de la composición de especies, la biomasa y la estructura de la comunidad (Ticktin 2004; Kumar y Shahabuddin, 2005). Esta información debe ser la base para guiar los criterios de aprovechamiento, no únicamente de vara sino de los PFNM en general. Es recomendable que los permisos de extracción tomen en cuenta indicadores de efectividad de las intervenciones, incluyendo medidas comparativas de la condición socio-ambiental antes y después del corte (Negi et al., 2011). Un análisis de mercado es también necesario, ya que los precios y la demanda de los PFNM es un componente importante para determinar la vulnerabilidad de las especies sometidas a cosecha (Oudendammer, 2006; TapiaTapia y Reyes-Chilpa, 2008).

Finalmente, es muy importante que la investigación de los aspectos mencionados sea conducida bajo un enfoque participativo que involucre a los dueños de los recursos. Como se documentó en este estudio, las percepciones de los sectores involucrados en el aprovechamiento de la vara sobre la capacidad de restablecimiento de la densidad de tallos después del corte dista mucho de lo que en realidad ocurre en el campo. Después de 12 años de trabajo en el ejido Campo Acosta, en Jalisco, la investigación participativa ha permitido un fortalecimiento en capacidades locales, traducidas en una revaloración del bosque como un medio que provee no solamente del recurso vara sino de diferentes servicios ecosistémicos. Dicho fortalecimiento se ha traducido en habilidades para el uso de instrumentos y metodologías de evaluación del bosque, así como en un incremento en la capacidad para la toma de decisiones internas al ejido relacionadas con el manejo del bosque. Por las bondades mencionadas para este tipo de investigación que ha probado ser exitosa para un aprovechamiento sostenible de PFNM en México (Peters, 2011), es recomendable que este enfoque sea extendido a otros ejidos sometidos a la extracción de estos recursos.

\section{Agradecimientos}

A los ejidatarios de la costa de Jalisco que tan generosamente nos han permitido trabajar en sus parcelas y apoyo de campo. PAPIIT-DGAPA, UNAM, y CONACYT brindaron el apoyo económico a través de los proyectos IN2246103 y No. 83441, respectivamente. La Estación de Biología Chamela, UNAM, facilitó la logística. Raúl Ahedo H., Enriquena Bustamante O., Salvador Araiza M. y Abel Verduzco, apoyaron con asistencia técnica y de campo. Agradecemos los comentarios de David Yetman y dos revisores anónimos que permitieron mejorar el trabajo. AMY y ABM trabajaron en el manuscrito durante una estancia sabática en la Universidad de Arizona, Tucson, y agradecen el apoyo de beca otorgada por el PASPA-DGAPA, UNAM.

\section{Literatura citada}

Anten N.P.R., Martínez-Ramos M. y Ackerly D. 2003. Defoliation and growth in an understory palm: quantifying the contributions of compensatory responses. Ecology 84:2905-2918.

Bowler D.E., Buyung-Ali L.M., Healey J.R., Jones J.P.G., Knight T.M. y Pullin A.S. 2012. Does community forest management provide global environmental benefits and improve local welfare? Frontiers in Ecology and the Environment 10:29-36.

Busby P.E., Vitousek P. y Dirzo R. 2010. Prevalence of tree regeneration by sprouting and seedling along a rainfall gradient in Hawaii. Biotropica 42:80-86.

CONABIO 2008. Capital Natural de México, vol. III: Políticas Públicas y Perspectivas de Sustentabilidad. Comisión Nacional para el Conocimiento y Uso de la Biodiversidad, México, D.F.

de Freitas J.V. y Pinard M.A. 2008. Applying ecological knowledge to decisions about seed tree retention in selective logging in tropical forests. Forest Ecology and Management 256:14341442.

FRA (Forest Resources Assessment) 2000. Non-wood forest products study for Mexico, Cuba and South America. Forest Resource Assessment Programme. FRA Working Paper 11. < fao.org/docrep/006/ac769e/ac769e00.htm> (consultado 26 febrero 2012).

FRA (Forest Resources Assessment) 2010. Global forest land use change from 1990 to 2005. <fao.org/forestry/fra/fra2010/en/> (consultado 26 febrero 2012).

Guevara S.A. 2000. Inversión ambiental y abatimiento de la pobreza: Evaluación económica y consideraciones de política pública. El caso del sur de Sonora. En: Muñoz V.C. y González M.A.C. Eds. Economía, Sociedad y Medio Ambiente. Reflexiones y Avances hacia un Desarrollo Sustentable en México, pp. 243-268, Instituto Nacional de Ecología, Secretaría del Medio Ambiente, Recursos Naturales y Pesca, México, D.F.

Kumar R. y Shahabuddin G. 2005. Effects of biomass extraction on vegetation structure, diversity and composition of forests in Sariska Tiger Reserve, India. Environmental Conservation 32:248-259.

Lemus-Vázquez J.A. y González-Guiza J.G. 1995. Diagnóstico dasonómico, ecológico y socioeconómico de las selvas del estado de Sinaloa. Tesis licenciatura, División de Ciencias Forestales, Universidad Autónoma Chapingo, Chapingo, Estado de México, 103 pp.

Lévesque M., McLaren K.P. y McDonald M.A. 2011. Coppice shoot dynamics in a tropical dry forest after human disturbance. Journal of Tropical Ecology 27:259-268.

Lindquist C.A. 1999. Economic necessity and ecological consequences the non-timber harvest of "vara blanca" in southern Sonora, Mexico. The Plant Press 23:9-11.

Lindquist C.A. 2000. Dimensions of sustainability: the use of vara blanca as a natural resource in the tropical deciduous forest of Sonora, Mexico. Tesis doctoral, Arid Lands Resource Sciences, The University of Arizona, Tucson, 211 pp. 
López-Toledo L., Anten N.P.R., Endress B.A., Ackerly D.D. y Martínez-Ramos M. 2012. Resilience to chronic defoliation in a dioecious understorey tropical rain forest palm. Journal of Ecology 100:1245-1256.

López-Urquidez G.A. 1997. Distribución y abundancia de la vara blanca (Croton spp.) en el estado de Sinaloa. Tesis maestría, Universidad Nacional Autónoma de México, Facultad de Ciencias, México, D.F., 107 pp.

Marshall E. y Newton A.C. 2003. Non-timber forest products in the community of El Terrero, Sierra de Manantlán Biosphere Reserve, México: is their use sustainable? Economic Botany 57:262-278.

Muro G.B.1987. El proceso de producción de vara blanca (Croton sp.) en el estado de Sinaloa. Memoria de seminario de titulación. División de Ciencias Forestales, Universidad Autónoma Chapingo, Chapingo, 80 pp.

Negi V.S., Maikhuri R.K. y Rawat L.S. 2011. Non-timber forest products (NTFPs): a viable option for biodiversity conservation and livelihood enhancement in central Himalaya. Biodiversity and Conservation 20:545-559.

Olmsted I. y Alvarez-Buylla E.R. 1995. Sustainable harvesting of tropical trees: demography and matrix models of two palm species in Mexico. Ecological Applications 5:484-500.

Oudendammer T.T. den. 2006. Non-timber forest products in the volcanic complex of Colima, Mexico. Reconnaissance study of the potential for NTFP extraction. Tesis maestría, Land use, Environment and Biodiversity, Utrecht University, Utrecht, 36 pp.

Palacios S.J.E., Mejía S.E., Oropeza M.J.L., Martínez M.M.R. y Figueroa S.B. 2009. Impacto de las actividades económicas en los recursos suelo y vegetación. TERRA Latinoamericana 27:247-255.

Pan Y., Richard A., Birdsey R.A., Fang J., Houghton R., Kauppi P.E., Kurz W.A., Phillips O.L., Shvidenko A., Lewis S.L., Canadell J.G., Ciais P., Jackson R.B., Pacala S.W., McGuire A.D., Piao S., Rautiainen A., Sitch S. y Hayes D. 2011. A large and persistent carbon sink in the world's forests. Science 333:988-993.

Peters C.M. 2011. Economic botany and management potential of
Neotropical seasonally dry forests. En: Dirzo R., Young H.S., Mooney H.A. y Ceballos G. Eds. Seasonally Dry Tropical Forests. Ecology and Conservation, pp. 239-257, Island Press, Washington, DC.

Rendón-Carmona H. 2002. Extracción de varas de Croton septemnervius McVaugh (Euphorbiaceae) y efecto del corte en su capacidad de rebrote en la costa de Jalisco, México. Tesis maestría, Universidad de Colima, Colima, 112 pp.

Rendón-Carmona H. 2011. Regeneración de especies leñosas sometidas a cosecha en un bosque tropical caducifolio de Jalisco: Elementos para su manejo. Tesis doctoral, Universidad Nacional Autónoma de México, México, D.F., 131 pp.

Rendón-Carmona H., Martínez-Yrízar A., Balvanera P. y PérezSalicrup D. 2009. Selective cutting of woody species in a Mexican tropical dry forest: Incompatibility between use and conservation. Forest Ecology and Management 257:567-579.

Reyes-Olivas A. 1993. Determinantes ecológicas de la "Vara Blanca" (Croton spp.) en el estado de Sinaloa. Tesis maestría, Colegio de Postgraduados, Montecillo, 85 pp.

Secretaría del Medio Ambiente, Recursos Naturales y Pesca (SEMARNAP). 1999. Atlas Forestal de México. SEMARNAP, Universidad Autónoma de Chapingo, Texcoco.

Tapia-Tapia E. C. y Reyes-Chilpa R. 2008. Productos forestales no maderables en México: aspectos económicos para el desarrollo sustentable. Madera y Bosques 14:95-112.

Ticktin, T. 2004. The ecological implications of harvesting nontimber forest products. Journal of Applied Ecology 41:11-21.

Weinbaum K.Z., Brashares J.S., Golden C.D. y Getz W.M. 2013. Searching for sustainability: are assessments of wildlife harvests behind the times? Ecology Letters 16: 99-111.

Yetman D.A., Van Devender T.R., López-Estudillo R.A. y Reina, A.L. 2000. Monte mojino: Mayo people and trees in Southern Sonora. En: Robichaux R.H., Yetman D.A. Eds. The Tropical Deciduous Forest of Alamos. Biodiversity of a Threatened Ecosystem in Mexico, pp. 102-151, University of Arizona Press, Tucson.

Recibido: 24 de noviembre de 2012

Aceptado: 11 de marzo de 2013 